\title{
ON NONNEGATIVE COSINE POLYNOMIALS WITH NONNEGATIVE INTEGRAL COEFFICIENTS
}

\author{
MIHAIL N. KOLOUNTZAKIS
}

(Communicated by J. Marshall Ash)

Dedicated to S. K. Pichorides

\begin{abstract}
We show that there exist $p_{0}>0$ and $p_{1}, \ldots, p_{N}$ nonnegative integers, such that

$$
0 \leq p(x)=p_{0}+p_{1} \cos x+\cdots+p_{N} \cos N x
$$

and $p_{0} \ll s^{1 / 3}$ for $s=\sum_{j=0}^{N} p_{j}$, improving on a result of Odlyzko who showed the existence of such a polynomial $p$ that satisfies $p_{0} \ll(s \log s)^{1 / 3}$. Our result implies an improvement of the best known estimate for a problem of Erdős and Szekeres. As our method is nonconstructive, we also give a method for constructing an infinite family of such polynomials, given one good "seed" polynomial.
\end{abstract}

\section{INTRODUCTION}

We consider nonnegative cosine polynomials of the form

$$
0 \leq p(x)=p_{0}+p_{1} \cos x+p_{2} \cos 2 x+\cdots+p_{N} \cos N x, \quad x \in[0,2 \pi],
$$

where $p_{j} \geq 0$. We also write $\widehat{p}(0)=p_{0}$. Notice that $p(0)=\sum_{j=0}^{N} p_{j}$ is the maximum of $p(x)$. We are interested in estimating the size of

$$
M(s)=\inf _{p(0) \geq s} \hat{p}(0)
$$

for $s \rightarrow \infty$. That is, we want to find polynomials of the above form for which $p_{0}=\frac{1}{2 \pi} \int_{0}^{2 \pi} p(x) d x$ is small compared to the maximum of $p(x)$. In what follows $C$ denotes an arbitrary positive constant and $a \ll b$ means $a \leq C b$ for some $C$.

If no more restrictions are imposed on the cosine polynomial $p(x)$ then $M(s)=0$ for all $s$. This is because the Fejér kernel

$$
K_{A}(x)=\sum_{j=-A}^{A}\left(1-\frac{|j|}{A+1}\right) e^{i j x}=1+\sum_{j=1}^{A} 2\left(1-\frac{j}{A+1}\right) \cos j x
$$

has constant coefficient 1 , has $K_{A}(0) \gg A$, and is nonnegative.

Received by the editors April 24, 1992.

1991 Mathematics Subject Classification. Primary 42A05, 42A61. 
If we restrict the coefficients $p_{1}, \ldots, p_{N}$ to be either 0 or 1 , we have the classical cosine problem, about which we know that for some $\varepsilon>0$

$$
2^{\log ^{\varepsilon} s} \ll M(s) \ll s^{1 / 2} .
$$

The upper bound in (1) is easily proved by considering the polynomial

$$
\begin{aligned}
f(x) & =\left(\sum_{1}^{A} \cos 3^{j} x\right)^{2} \\
& =A+\frac{1}{2} \sum_{j=1}^{A} \cos \left(2 \cdot 3^{j} x\right)+\sum_{\substack{k, l=1 \\
k>l}}^{A}\left(\cos \left(3^{k}+3^{l}\right) x+\cos \left(3^{k}-3^{l}\right) x\right) .
\end{aligned}
$$

All cosines in (3) have distinct frequencies. Define $f_{1}(x)=f(x)+\frac{1}{2} A-$ $\frac{1}{2} \sum_{j=1}^{A} \cos \left(2 \cdot 3^{j} x\right)$. Then $f_{1}(x) \geq 0, f_{1}(0) \gg A^{2}, \widehat{f}_{1}(0) \ll A$, and $f_{1}$ has nonconstant coefficients which are either 0 or 1 . The lower bound in (1) is much harder to prove and is due to Bourgain [3]. Earlier, Roth [8] had obtained $M(s) \gg(\log s / \log \log s)^{1 / 2}$.

From this point on, we will study the case of $p_{1}, \ldots, p_{N}$ being arbitrary nonnegative integers. This case was studied by Odlyzko [7] who showed that

$$
M(s) \ll(s \log s)^{1 / 3} .
$$

The method is the following. Consider the nonnegative polynomial

$$
q(x)=\alpha K_{A}(x)=q_{0}+q_{1} \cos x+\cdots+q_{A} \cos A x,
$$

whose coefficients are not necessarily integers $(\alpha>0)$. We modify $q$ so that its nonconstant coefficients are integers, by adding to it a random polynomial

$$
r(x)=r_{1} \cos x+\cdots+r_{A} \cos A x .
$$

The coefficients $r_{j}$ are independent random variables which take values such that $r_{j}+q_{j}$ is always an integer. A theorem of Salem and Zygmund [9] guarantees that $\|r\|_{\infty}$ is small with high probability, and the nonnegative polynomial $p(x)=q(x)+r(x)+\|r\|_{\infty}$ achieves (4) when $\alpha$ is appropriately chosen as a function of $A$.

Odlyzko studied this problem in connection with a problem posed by Erdős and Szekeres [4]. The problem is to estimate

$$
E(n)=\inf \max _{|z|=1}\left|\prod_{k=1}^{n}\left(1-z^{a_{k}}\right)\right|
$$

where $a_{1}, \ldots, a_{n}$ may be any positive integers. The inequality

$$
\log E(n) \ll M(n) \log (n)
$$

holds (see [7]), so that Odlyzko's result implies $\log E(n) \ll n^{1 / 3} \log ^{4 / 3} n$.

In this paper we replace the random modification in Odlyzko's argument with a more careful modification, based, again, partly on randomization. We use a recent theorem of Spencer [10] which in some cases does better than the SalemZygmund theorem. We show in $\S 3$ that, when $p_{1}, \ldots, p_{N}$ are restricted to be 
nonnegative integers, we have $M(s) \ll s^{1 / 3}$. By (5) this implies $\log E(n) \ll$ $n^{1 / 3} \log n$. Our method is similar to that used by Beck [2] on a different problem, posed by Littlewood. ${ }^{1}$

Both the Salem-Zygmund theorem and Spencer theorem are nonconstructive. In $\S 4$ we give a deterministic procedure which, given a polynomial $p(x)$ with nonnegative integral Fourier coefficients (in other words, $p_{j}$ is a nonnegative even integer, for $j \geq 1)$ and with $\widehat{p}(0) \leq(p(0))^{\alpha}$, for some $\alpha>0$, produces a sequence of polynomials $p=p^{(0)}, p^{(1)}, p^{(2)}, \ldots$, such that $\operatorname{deg} p^{(n)} \rightarrow \infty$, $p^{(n)}(0) \rightarrow \infty$, and $\left(p^{(n)}\right)^{\Upsilon}(0) \leq\left(p^{(n)}(0)\right)^{\alpha}$. This shows $M(s) \leq C s^{1 / \alpha}$, with $C$ dependent on the initial $p$ only.

\section{BOUNDS ON RANDOM TRIGONOMETRIC POLYNOMIALS}

In [7] the following classical theorem was used to estimate the size of a random polynomial.

Theorem 1 (Salem and Zygmund [9; 5, p. 69]). Let $f_{1}(x), \ldots, f_{n}(x)$ be trigonometric polynomials of degree at most $m$ and $\xi_{1}, \ldots, \xi_{n}$ be independent random variables, which satisfy $\mathbf{E} e^{\lambda \xi_{j}} \leq e^{\lambda^{2} / 2}$ for all $j$ and $\lambda>0$ (subnormal random variables). Write

$$
f(x)=\sum_{j=1}^{n} \xi_{j} f_{j}(x) .
$$

Then, for some $C>0$,

$$
\operatorname{Pr}\left(\|f\|_{\infty} \geq C\left(\sum_{j=1}^{n}\left\|f_{j}\right\|_{\infty}^{2} \log m\right)^{1 / 2}\right) \leq \frac{1}{m^{2}} .
$$

Theorem 1 was used in [7] to change the coefficients of a polynomial to integers without a big loss:

Corollary 1. Let $p(x)=p_{0}+\sum_{j=1}^{N} p_{j} \cos j x$ and define the random polynomial $r(x)$ so that $p(x)+r(x)$ always has integral coefficients (except perhaps the constant coefficient):

$$
r(x)=\sum_{j=1}^{N} \xi_{j} \cos j x
$$

with $\xi_{j}=0$ if $p_{j}$ is an integer, else

$$
\xi_{j}= \begin{cases}\left\lfloor p_{j}\right\rfloor-p_{j} & \text { with probability }\left\lceil p_{j}\right\rceil-p_{j}, \\ \left\lceil p_{j}\right\rceil-p_{j} & \text { with probability } p_{j}-\left\lfloor p_{j}\right\rfloor .\end{cases}
$$

Then $\operatorname{Pr}\left(\|r\|_{\infty} \ll(N \log N)^{1 / 2}\right) \rightarrow 1$, as $N \rightarrow \infty$.

Proof of Corollary 1. The above defined $\xi_{j}$ are subnormal (see, e.g., [1, p. 235, Lemma A.6]). Theorem 1 can now be applied.

\footnotetext{
${ }^{1}$ After this paper was submitted for publication the author learned that the method employed for the proof of the basic result has also appeared in [6].
} 
The following theorem of Spencer [10] is sometimes better than the SalemZygmund theorem, though unfortunately only in the symmetric case $\xi_{j}= \pm 1$ (Rademacher random variables).

Theorem 2 (Spencer [10]). Let $a_{i j}, i=1, \ldots, n_{1}, j=1, \ldots, n_{2}$, be such that $\left|a_{i j}\right| \leq 1$. Then there are signs $\varepsilon_{1}, \ldots, \varepsilon_{n_{2}} \in\{-1,1\}$ such that, for all $i$,

$$
\left|\sum_{j=1}^{n_{2}} \varepsilon_{j} a_{i j}\right| \leq C n_{1}^{1 / 2} \text {. }
$$

Notice there is no dependence of the bound on $n_{2}$.

Corollary 2. Let $f_{1}(x), \ldots, f_{n}(x),\left\|f_{j}\right\|_{\infty} \leq C$, be trigonometric polynomials of degree at most $m$. Then there is a choice of signs $\varepsilon_{1}, \ldots, \varepsilon_{n} \in\{-1,1\}$ such that

$$
\left\|\sum_{j=1}^{n} \varepsilon_{j} f_{j}\right\|_{\infty} \leq C m^{1 / 2}
$$

Proof of Corollary 2. For $i=1, \ldots, 10 m, j=1, \ldots, n$, define $a_{i j}=f_{j}\left(x_{i}\right)$, where $x_{i}=i \frac{2 \pi}{10 m}$. Let $\varepsilon_{1}, \ldots, \varepsilon_{n}$ be the sequence of signs given by Theorem 2 for the matrix $a_{i j}$ and write $f=\sum_{j=1}^{n} \varepsilon_{j} f_{j}$. There is $x_{0} \in[0,2 \pi]$ such that $\left|f\left(x_{0}\right)\right|=\|f\|_{\infty}$. For some $k$ we have $\left|x_{k}-x_{0}\right| \leq \frac{2 \pi}{10 m}$. By Bernstein's inequality, $\left\|f^{\prime}\right\|_{\infty} \leq m\|f\|_{\infty}$, we get

$$
\left|f\left(x_{0}\right)-f\left(x_{k}\right)\right| \leq \frac{2 \pi}{10 m}\left\|f^{\prime}\right\|_{\infty} \leq \frac{2 \pi}{10}\left|f\left(x_{0}\right)\right|
$$

which, since $\frac{2 \pi}{10}<1$, implies

$$
\|f\|_{\infty}=\left|f\left(x_{0}\right)\right| \leq C\left|f\left(x_{k}\right)\right|=C\left|\sum_{j=1}^{n} \varepsilon_{j} a_{k j}\right| \leq C m^{1 / 2},
$$

and the proof is complete.

Corollary 2 is better than Theorem 1 only when $m / \log m=o(n)$. It is a strictly symmetric result and cannot directly be applied to modify a polynomial $p(x)$ so that it has integral coefficients, as we need to do in our case. We show in $\S 3$ that a sequence of applications of Corollary 2 is needed.

\section{Proof of the Inequality $M(s) \ll s^{1 / 3}$}

Since Corollary 2 only allows us to choose random signs, we cannot use it directly (as we used the Salem-Zygmund theorem) to modify the coefficients of a polynomial to integers, while controlling the size of the change. In this section we show how to modify the coefficients little by little to achieve the same result.

Let $\alpha>0$ and define

$$
a(x)=\alpha K_{A}(x)=\sum_{j=0}^{A} a_{j} \cos j x .
$$


Suppose $\varepsilon>0$ and the nonnegative integer $k_{0}$ is such that for some nonnegative integers $b_{j}$

$$
\left|a_{j}-b_{j} 2^{-k_{0}}\right| \leq \varepsilon \text { for all } j=1, \ldots, A .
$$

We shall define a finite sequence of polynomials

$$
a^{(0)}(x)=a_{0}+\sum_{j=1}^{A} b_{j} 2^{-k_{0}} \cos j x, a^{(1)}(x), \ldots, a^{\left(k_{0}\right)}(x)
$$

inductively, so that if $a^{(k)}(x)=a_{0}+\sum_{j=1}^{A} a_{j}^{(k)} \cos j x$ then, for each $j=$ $1, \ldots, A$,

$$
a_{j}^{(k)}=b_{j}^{(k)} 2^{k-k_{0}}
$$

for some nonnegative integers $b_{j}^{(k)}$. We define inductively the coefficients of $a^{(k+1)}$ as follows. If $b_{j}^{(k)}, j>0$, is even then $a_{j}^{(k+1)}=a_{j}^{(k)}$. Else define

$$
a_{j}^{(k+1)}=a_{j}^{(k)}+\varepsilon_{j}^{(k)} 2^{k-k_{0}}
$$

where $\varepsilon_{j}^{(k)} \in\{-1,1\}$ are such that

$$
\left\|\sum_{b_{j}^{(k)} \text { odd }} \varepsilon_{j}^{(k)} \cos j x\right\| \leq C A^{1 / 2} .
$$

The existence of the signs $\varepsilon_{j}^{(k)}$ is guaranteed by Corollary 2. Notice that (8) implies the preservation of $(7)$ by the inductive definition. We deduce from (9) that

$$
\left\|a^{(k+1)}-a^{(k)}\right\|_{\infty} \leq C 2^{k-k_{0}} A^{1 / 2} .
$$

The polynomial $a^{\left(k_{0}\right)}$ has integral coefficients (except perhaps for the constant coefficient). Summing (10) we get

$$
\left\|a-a^{\left(k_{0}\right)}\right\|_{\infty} \leq\left\|a-a^{(0)}\right\|_{\infty}+\left\|a^{(0)}-a^{\left(k_{0}\right)}\right\|_{\infty} \leq A \varepsilon+C A^{1 / 2}
$$

Choose $\varepsilon=1 / A$ to get $\left\|a-a^{\left(k_{0}\right)}\right\|_{\infty} \leq C A^{1 / 2}$. On the other hand, the coefficients of $a$ and $a^{\left(k_{0}\right)}$ differ by at most 1 , and this implies that for the nonnegative polynomial $p(x)=a^{\left(k_{0}\right)}(x)+\left\|a-a^{\left(k_{0}\right)}\right\|_{\infty}$ we have

$$
\begin{gathered}
p(0) \geq a(0)-A \geq C \alpha A-A, \\
\widehat{p}(0)=\alpha+\left\|a-a^{\left(k_{0}\right)}\right\|_{\infty} \leq \alpha+C A^{1 / 2} .
\end{gathered}
$$

Select $\alpha=A^{1 / 2}$ to get $\widehat{p}(0) \ll A^{1 / 2}$ and $p(0) \gg A^{3 / 2}$. Since $p$ has integral coefficients, we have exhibited a polynomial that achieves $M(s) \ll s^{1 / 3}$, and the proof is complete.

Remark on cosine sums. Applying the method of the preceding proof on the coefficients of the Fejér kernel $K_{A}(x)$, one ends up with a nonnegative polynomial 
of degree at most $A$, which is of the form

$$
p(x)=p_{0}+2 \sum_{j=1}^{k} \cos \lambda_{j} x
$$

where $\lambda_{j} \in\{1, \ldots, A\}$ are distinct. We have $\left\|K_{A}-p\right\|_{\infty} \ll A^{1 / 2}$ which, since $p_{0}=\frac{1}{2 \pi} \int_{0}^{2 \pi} p(x) d x$, implies

$$
p_{0} \ll A^{1 / 2} \text { and } p(0) \gg A \text {. }
$$

Thus $p$ is a new example of a cosine sum that achieves the upper bound in (1). It is not as simple as the one mentioned in the introduction but the spectrum of it is much denser: $\frac{1}{2} A+O\left(A^{1 / 2}\right)$ cosines with frequencies from 1 to $A$.

Since the Dirichlet kernel

$$
\begin{aligned}
D_{A}(x) & =\sum_{j=-A}^{A} e^{i j x} \\
& =1+2 \sum_{j=1}^{A} \cos j x \\
& =\frac{\sin \left(A+\frac{1}{2}\right) x}{\sin \frac{x}{2}}
\end{aligned}
$$

has a minimum asymptotically equal to $-\frac{4}{3 \pi} A$, it is conceivable that one may be able to raise the above number of cosine from $\frac{1}{2} A+O\left(A^{1 / 2}\right)$ to

$$
\left(1-\frac{2}{3 \pi}\right) A+o(A) \text {. }
$$

In other words, since

$$
\min _{x} \sum_{j=1}^{A} \cos j x=-\frac{2}{3 \pi} A+o(A),
$$

one must remove at least $\frac{2}{3 \pi} A$ cosines from the above sum in order to make its minimum $O(A)$ in absolute value.

Note added in proof. The author has now proved that $\frac{1}{2} A+O(A)$ is best possible.

\section{THE CONSTRUCTION}

Suppose we are given a polynomial $p(x) \geq 0$ of degree $d$, whose nonconstant coefficients are even nonnegative integers, which satisfies

$$
\widehat{p}(0) \leq(p(0))^{\alpha}
$$

for some $\alpha>0$. Define the infinite sequence of nonnegative polynomials $p=p^{(1)}, p^{(2)}, p^{(3)}, \ldots$, with the recursive formula

$$
p^{(k+1)}(x)=p^{(k)}((d+1) x) \cdot p(x) .
$$


Since $p$ has even nonconstant coefficients, the Fourier coefficients of all $p^{(k)}$ are nonnegative integers. The spectrum of the first factor in (16) is supported by the multiples of $d+1$, and that of the second factor is supported by the interval $[-d, d]$. This implies that $\left(p^{(k+1)}\right)^{\curlyvee}(0)=\left(p^{(k)}\right)^{\curlyvee}(0) \widehat{p}(0)$. We obviously have $p^{(k+1)}(0)=p^{(k)}(0) p(0)$. We conclude that for all $k \geq 0$

$$
\left(p^{(k)}\right)^{-}(0)=(\widehat{p}(0))^{k} \quad \text { and } \quad p^{(k)}(0)=(p(0))^{k},
$$

and consequently

$$
\left(p^{(k)}\right)^{\Upsilon}(0) \leq\left(p^{(k)}(0)\right)^{\alpha} .
$$

So, if $s$ is a power of $p(0)$, we have $M(s) \leq s^{\alpha}$, and for any $s$ we have $M(s) \leq C s^{\alpha}$, where $C=(p(0))^{\alpha}$.

As an example we give

$$
p(x)=4+4 \cos x+2 \sum_{j=2}^{10} \cos j x
$$

which can be checked numerically to be positive and has constant coefficient $\widehat{p}(0)=4$ and $p(0)=26$. This gives $\alpha=\log 4 / \log 26=0.42549 \ldots$.

In view of the above construction, finding a single polynomial $p$ with $\widehat{p}(0) \leq$ $(p(0))^{\alpha}$, with $\alpha<\frac{1}{3}$, will prove that the result in this paper is not the best possible. The above example was actually found by a computer, but if no more insight is gained into how these good "seed" polynomials look, the computing time grows dramatically as we increase the degree of the polynomial.

\section{ACKNOWLEDGMENT}

I would like to thank P. J. Cohen and A. M. Odlyzko for their help.

\section{REFERENCES}

1. N. Alon and J. Spencer, The probabilistic method, Wiley Interscience Series in Discrete Mathematics and Optimization, 1992.

2. J. Beck, Flat polynomials on the unit circle-Note on a problem of Littlewood, Bull. London Math. Soc. 23 (1991), 269-277.

3. J. Bourgain, Sur le minimum d'une somme de cosinus, Acta Arith. 45 (1986), 381-389.

4. P. Erdős and G. Szekeres, On the product $\prod_{k=1}^{n}\left(1-z^{a_{k}}\right)$, Acad. Serbe Sci. Publ. Inst. Math. 13 (1959), 29-34.

5. J.-P. Kahane, Some random series of functions, 2nd ed., Cambridge Stud. Adv. Math., vol. 5, Cambridge Univ. Press, Cambridge and New York, 1985.

6. L. Lovász, J. Spencer, and K. Vesztergombi, Discrepancy of set systems and matrices, European J. Combin. 7 (1986), 151-160.

7. A. M. Odlyzko, Minima of cosine sums and maxima of polynomials on the unit circle, $\mathrm{J}$. London Math. Soc. (2) 26 (1982), 412-420.

8. K. F. Roth, On cosine polynomials corresponding to sets of integers, Acta Arith. 24 (1973), 347-355.

9. R. Salem and A. Zygmund, Some properties of trigonometric series whose terms have random signs, Acta Math. 91 (1954), 245-301

10. J. Spencer, Six standard deviations suffice, Trans. Amer. Math. Soc. 289 (1985), 679-706.

Department of Mathematics, Stanford University, Stanford, California 94305

E-mail address: kolount@cauchy.stanford.edu 